\title{
Transformation of a copula using the associated co-copula
}

https://doi.org/10.1515/demo-2018-0017

Received September 26, 2018; accepted November 1, 2018

Abstract: We investigate the properties of a new transformation of copulas based on the co-copula and an univariate function. It is shown that several families in the copula literature can be interpreted as particular outputs of this transformation. Symmetry, association, ordering and dependence properties of the resulting copula are established.

Keywords: Copula, co-copula

MSC: $62 \mathrm{H} 05$

\section{Introduction}

Let $\mathcal{C}$ be the set of all bivariate copulas. For all function $f:[0,1] \rightarrow[0,1], C \in \mathcal{C}$ and $(u, v) \in[0,1]^{2}$, introduce the following transformation of a bivariate copula:

$$
T(f, C)(u, v)=C(u, v) f\left(C^{\star}(u, v)\right),
$$

where $C^{\star}$ is the co-copula associated with $C$, i.e.

$$
C^{\star}(u, v)=u+v-C(u, v)=\mathbb{P}(U \leq u \cup V \leq v),
$$

the random pair $(U, V)$ being distributed from the copula $C(u, v)=\mathbb{P}(U \leq u \cap V \leq v)$. Let us stress that $T(f, C)$ may not be a copula for any function $f$ and copula $C$. We thus shall investigate conditions on $f$ such that $T(f, C)$ is a copula for any copula $C$ : In the following, a function $f$ is said to be admissible if and only if $T(f, C) \in \mathcal{C}$ for all $C \in \mathcal{C}$.

Several particular cases of this transformation have been investigated in the copula literature. Four parametric models for $f$ combined with arbitrary copulas $C$ have been considered in [5, 7] while, conversely, [6, 9] and [1], Paragraph 5.1, considered an arbitrary function with a specific copula, see Section 2 for details. In this work, we study the transformation in its full generality: Both the function $f$ and the copula $C$ may be arbitrary.

A number of recent works are dedicated to the transformation of copulas: $[8,10,14,17,20]$, while $[4,16]$ focused on Archimedean copulas. The usual motivation is that, starting from a simple parametric family, the transformation may lead to a new copula with increased flexibility. Finally, we refer to $[11,18]$ for nice introductions to copula theory.

The goal of Section 2 is to characterize the set of admissible functions and to provide some examples. The symmetry and ordering properties of the transformed copula are established in Section 3 and Section 4 respectively. The dependence aspects are studied in Section 5: The consequences of the transformation on tail dependence, association measures and dependence properties are exhibited.

^Corresponding Author: Stéphane Girard: Univ. Grenoble Alpes, Inria, CNRS, Grenoble INP, LJK, 38000 Grenoble, France 


\section{Admissible functions}

In Subsection 2.1, we establish necessary and sufficient conditions on a function $f$ to be admissible i.e. so that $T(f, C)$ is still a copula whatever the starting copula $C$. Then, some properties of admissible functions are derived in Subsection 2.2.

\subsection{Necessary and sufficient conditions}

For all $(u, v) \in[0,1]^{2}$, let $W(u, v)=\max (u+v-1,0)$ and $M(u, v)=\min (u, v)$ be the two FréchetHoeffding bounds. Let also introduce $\Pi(u, v)=u v$ the independence copula. Remark that the property $W(u, v) \leq C(u, v) \leq M(u, v)$ for all $C \in \mathcal{C}$ (see for instance (2.2.5) in [18]) entails $0 \leq u+v-C(u, v) \leq 1$ and therefore the transformed copula (1) is well-defined for all $(u, v) \in[0,1]^{2}$ and $f:[0,1] \rightarrow[0,1]$.

Our first result provides necessary and sufficient conditions so that the margins of $T(f, C)$ are uniform. Its proof is straightforward.

Lemma 1 (Boundary conditions).

(i) For all function $f$, one has $T(f, C)(u, 0)=T(f, C)(0, u)=0$ for all $u \in[0,1]$.

(ii) A necessary and sufficient condition for $T(f, C)(u, 1)=T(f, C)(1, u)=u$ for all $u \in[0,1]$ is $f(1)=1$.

The second step is to investigate the effect of the mapping $T(f, \cdot)$ on quasi-copulas. Recall that, from [11, Chapter 7], a quasi-copula $Q$ satisfies the same boundary, increasing and Lipschitz properties

$$
\left|Q\left(u_{2}, v_{2}\right)-Q\left(u_{1}, v_{1}\right)\right| \leq\left|u_{2}-u_{1}\right|+\left|v_{2}-v_{1}\right| \text { for all }\left(u_{1}, u_{2}, v_{1}, v_{2}\right) \in[0,1]^{4},
$$

(see [18, Theorem 2.2.4]) as do copulas but is not necessarily 2-increasing. Let us recall that, in (1), the mapping $T(f, \cdot)$ is defined on $\mathcal{C}$. The mapping can yet be applied to quasi-copulas by formally extending $T(f, \cdot)$ to the set functions $H:[0,1]^{2} \rightarrow[0,1]$ that satisfy $W(u, v) \leq H(u, v) \leq M(u, v)$ for all $(u, v) \in[0,1]^{2}$.

Proposition 1 (Transformation of quasi-copula). If $Q$ is a quasi-copula, $f$ is positive, increasing, 1 -Lipschitzian and $f(1)=1$ then $T(f, Q)$ is a quasi-copula.

Proof. Let us consider the expansion:

$$
\begin{aligned}
T(f, Q)(u+h, v)-T(f, Q)(u, v) & =\Delta_{1}(u, v, h)+\Delta_{2}(u, v, h), \\
\Delta_{1}(u, v, h) & =(Q(u+h, v)-Q(u, v)) f(u+h+v-Q(u+h, v)), \\
\Delta_{2}(u, v, h) & =Q(u, v)(f(u+h+v-Q(u+h, v))-f(u+v-Q(u, v))) .
\end{aligned}
$$

Since $f$ is positive, increasing and $f(1)=1$, it follows that $0 \leq f(t) \leq 1$ for all $t \in[0,1]$ and thus

$$
0 \leq \Delta_{1}(u, v, h) \leq Q(u+h, v)-Q(u, v) .
$$

Moreover, $Q$ is a quasi-copula implies that $Q(\cdot, v)$ is increasing and 1-Lipschitzian (see (2)), so that

$$
(u+h+v-Q(u+h, v))-(u+v-Q(u, v))=h+Q(u, v)-Q(u+h, v) \geq 0 .
$$

Therefore, in view of the Lipschitz and increasing properties of $f$,

$$
0 \leq f(u+h+v-Q(u+h, v))-f(u+v-Q(u, v)) \leq h+Q(u, v)-Q(u+h, v),
$$

which entails

$$
0 \leq \Delta_{2}(u, v, h) \leq h-Q(u+h, v)+Q(u, v) .
$$

Collecting (3) and (4) yields $0 \leq T(f, Q)(u+h, v)-T(f, Q)(u, v) \leq h$ i.e. $T(f, Q(\cdot, v))$ is increasing and 1-Lipschitzian. The proof that $T(f, Q)(u, \cdot)$ is increasing and 1-Lipschitzian is similar. Finally, from Lemma 1, the boundary conditions are fulfilled and therefore $T(f, Q)$ is a quasi-copula. 
Let us consider $\mathcal{D}$ the set of increasing functions $f$ such that $f(1)=1$ and $I_{d} / f$ is increasing where $I_{d}$ denotes the identity function. The effect of the mapping $T(f, \cdot)$ on the Fréchet-Hoeffding lower and upper bounds is illustrated in the next lemma:

Lemma 2 (Fréchet-Hoeffding bounds).

(i) $T(f, W)=W$ if and only if $f(1)=1$.

(ii) For all function $f:[0,1] \rightarrow[0,1], T(f, M)=C_{f}$ where

$$
C_{f}(u, v)=\min (u, v) f(\max (u, v)),(u, v) \in[0,1]^{2} .
$$

Moreover, if $f$ is continuous, differentiable at all but at most countably many points, then $C_{f} \in \mathcal{C}$ if and only if $f \in \mathcal{D}$.

Proof. The proof of (i) is straightforward while (ii) was proved in [6, Theorem 1]. In particular, the condition that $I_{d} / f$ is increasing is proved to be necessary in [6, Lemma 1].

It appears that, provided $f(1)=1$, the Fréchet-Hoeffding lower bound $W$ is a fixed point of $T(f, \cdot)$. Besides, the new class of symmetric bivariate copulas (5) introduced in [6], can be interpreted as the transformation of the Fréchet-Hoeffding upper bound $M$ by the mapping $T(f, \cdot)$. Let $\mathcal{F}$ the set of increasing, continuous, convex functions $f:[0,1] \rightarrow[0,1]$ such that $f(1)=1$ and $I_{d} / f$ is increasing. Our main result is the following:

Theorem 1 (Necessary and sufficient conditions). $f$ is admissible if and only if $f \in \mathcal{F}$.

Proof. Let us first assume that $f$ is admissible. Then, following Lemma 2(i), $T(f, M)(u, v)=C_{f}(u, v)=u f(v)$ for all $0 \leq u \leq v \leq 1$. Therefore, since $C_{f}$ is a copula, $f$ is necessarily continuous on $[0,1]$. Let $0<t_{1}<$ $t_{2} \leq 1$ and consider $C_{1}$ the copula defined as the ordinal sum of one element with respect to [ $\left.t_{1}, t_{2}\right]$, see [11, Section 3.2.2] for a detailed account on ordinal sums. Since $\tilde{C}_{1}:=T\left(f, C_{1}\right)$ is a copula, the associated $\tilde{C}_{1}$ volume of $\left[t_{1},\left(t_{1}+t_{2}\right) / 2\right]$ is positive:

$$
\begin{gathered}
\tilde{C}_{1}\left(\left(t_{1}+t_{2}\right) / 2,\left(t_{1}+t_{2}\right) / 2\right)-\tilde{C}_{1}\left(\left(t_{1}+t_{2}\right) / 2, t_{1}\right)-\tilde{C}_{1}\left(t_{1},\left(t_{1}+t_{2}\right) / 2\right)+\tilde{C}_{1}\left(t_{1}, t_{1}\right) \\
=t_{1}\left(f\left(t_{1}\right)+f\left(t_{2}\right)-2 f\left(\left(t_{1}+t_{2}\right) / 2\right)\right) \geq 0 .
\end{gathered}
$$

This shows that $f$ is mid-point convex. Recall that $f$ is continuous, this implies that $f$ is convex which in turn implies that $f$ is differentiable at all but at most countably many points. Lemma 2(ii) then yields that $f \in \mathcal{D}$, in particular $f$ is increasing $f, I_{d} / f$ is increasing and $f(1)=1$. As a conclusion, $f \in \mathcal{F}$.

Conversely, assume that $f \in \mathcal{F}$. The proof that $f$ is admissible follows the same lines as the one of [7, Proposition 2.2]. Let us first assume that $C$ is an absolutely continuous copula with continuous mixed partial derivatives denoted by $D_{1} C:=\partial C / \partial u, D_{2} C:=\partial C / \partial v$ and $D_{12} C:=\partial^{2} C / \partial u \partial v$. Let us also assume that $f$ is twice differentiable. Then, $I_{d} / f$ is increasing implies $f(t)-t f^{\prime}(t) \geq 0$ for all $t \in[0,1]$ and therefore $f^{\prime}(1) \leq 1$. Now, $f^{\prime \prime}>0$ implies that $f^{\prime}$ is increasing so that $f^{\prime}(t) \leq f^{\prime}(1) \leq 1$ for all $t \in[0,1]$. This proves that $f$ is 1 -Lipschitzian and, from Proposition $1, T(f, C)$ is a quasi-copula. To check the 2-increasing property, consider the density:

$$
\begin{aligned}
D_{12} T(f, C) & =C D_{1} C^{\star} D_{2} C^{\star} f^{\prime \prime}\left(C^{\star}\right) \\
& +f^{\prime}\left(C^{\star}\right)\left[\left(1-D_{1} C^{\star}\right) D_{2} C^{\star}+\left(1-D_{2} C^{\star}\right) D_{1} C^{\star}\right] \\
& +D_{12} C\left[f\left(C^{\star}\right)-C f^{\prime}\left(C^{\star}\right)\right] .
\end{aligned}
$$

This expansion can be rewritten equivalently as

$$
\begin{aligned}
D_{12} T(f, C) & =C D_{1} C^{\star} D_{2} C^{\star} f^{\prime \prime}\left(C^{\star}\right)+D_{12} C\left(C^{\star}-C\right) f^{\prime}\left(C^{\star}\right) \\
& +f^{\prime}\left(C^{\star}\right)\left[\left(1-D_{1} C^{\star}\right) D_{2} C^{\star}+\left(1-D_{2} C^{\star}\right) D_{1} C^{\star}\right] \\
& +D_{12} C\left[f\left(C^{\star}\right)-C^{\star} f^{\prime}\left(C^{\star}\right)\right] .
\end{aligned}
$$


Recall that $0 \leq D_{1} C^{\star} \leq 1$ and $0 \leq D_{2} C^{\star} \leq 1$ from [18, Theorem 2.2.7]. It is thus clear that (7) is non-negative because $f$ is increasing. Besides, $f\left(C^{\star}\right)-C^{\star} f^{\prime}\left(C^{\star}\right) \geq 0$ since $I_{d} / f$ is increasing and thus (8) is non-negative. Finally, $C^{\star}(u, v)-C(u, v)=u+v-2 C(u, v) \geq 0$ for all $(u, v) \in[0,1]^{2}$ in view of $C(u, v) \leq M(u, v) \leq(u+v) / 2$. Assuming $f^{\prime \prime} \geq 0$ implies that (6) is also non-negative. As a first conclusion, if $C$ is an absolutely continuous copula with continuous mixed partial derivatives and $f$ is twice differentiable, then $T(f, C)$ is a copula.

Second, let us consider the case of an arbitrary copula $C$ in $\mathcal{C}$ and arbitrary function $f$ in $\mathcal{F}$. Recall that copulas with continuous mixed partial derivatives are dense in $\mathcal{C}$ and that twice differentiable convex functions are dense in the set of convex functions, both with respect to the $L_{\infty}$ norm. It is easily seen that $T(f, \cdot)$ and $T(\cdot, C)$ are continuous with respect to the $L_{\infty}$ norm. Therefore, by density, $T(f, C)$ is a copula for any $C \in \mathcal{C}$ and $f \in \mathcal{F}$.

Remark 1. In [15], a similar mapping based on the joint survival function $1-C^{\star}$ is investigated. The necessary and sufficient conditions of Theorem 1 can thus be related to the ones provided in [15, Theorem 2] by considering $f(1-\cdot)$.

\subsection{First properties}

The next result exhibits some properties of admissible functions. First, the identity is the only admissible function vanishing at the origin. Second, $I_{d} \in \mathcal{F}$ and the stability of $\mathcal{F}$ with respect to composition entail that $\mathcal{F}$ is a sub-monoid of the set of functions defined on the unit interval. Besides, the stability of $\mathcal{F}$ with respect to convex combinations shows that $\mathcal{F}$ is a convex set. Third, any admissible function is either constant or strictly increasing in a neighbourhood of 1 . This property will reveal useful in Section 4.

Lemma 3 (Properties of admissible functions).

(i) If $f \in \mathcal{F}$ then $t \leq f(t) \leq 1$ for all $t \in[0,1]$.

(ii) If $f \in \mathcal{F}$ and $f(0)=0$, then $f=I_{d}$.

(iii) Let $(f, g) \in \mathcal{F}^{2}$ and $p \in[0,1]$. Then, $f \circ g \in \mathcal{F}$ and $p f+(1-p) g \in \mathcal{F}$.

(iv) Let $f \in \mathcal{F}$. Then, either $f \equiv 1$ or there exists $x_{0} \in(0,1)$ such that $f$ is strictly increasing on $\left[x_{0}, 1\right]$.

Proof. (i) The property $t \leq f(t)$ is a consequence of $I_{d} / f$ is increasing and $f(1)=1$ while $f(t) \leq 1$ is implied by $f$ is increasing and $f(1)=1$.

(ii) Let $f \in \mathcal{F}$. From [3, Theorem 5], $f$ is convex and $f(0)=0$ imply that $f$ is star-shaped i.e. $f / I_{d}$ is increasing. Taking account of $f \in \mathcal{F}$ leads to $f=c I_{d}$ where $c \in \mathbb{R}$. Since $f(1)=1$, we obtain $c=1$ and the conclusion follows.

(iii) Let $(f, g) \in \mathcal{F}^{2}$. Remarking that $I_{d} / f \circ g=\left(I_{d} / f\right) \times(f / f \circ g)$, it is clear that $f \circ g \in \mathcal{F}$. The proof of $p f+(1-p) g \in \mathcal{F}$ for all $p \in[0,1]$ is straightforward.

(iv) Let $f \in \mathcal{F}$. First, if $f$ is constant then, necessarily $f \equiv 1$ in view of the condition $f(1)=1$. Second, if $f$ is not constant, assume that, for all $x_{0} \in(0,1)$, there exists $x_{0}<x_{1}<x_{2}$ such that $f\left(x_{0}\right)<f\left(x_{1}\right)=f\left(x_{2}\right)$. Let $p \in(0,1)$ such that $p x_{0}+(1-p) x_{2}=x_{1}$. The convexity property $f\left(p x_{0}+(1-p) x_{2}\right) \leq p f\left(x_{0}\right)+(1-p) f\left(x_{2}\right)$ can be rewritten as $f\left(x_{1}\right) \leq p f\left(x_{0}\right)+(1-p) f\left(x_{1}\right)$ or equivalently $f\left(x_{1}\right) \leq f\left(x_{0}\right)$. This contradicts the assumption $f\left(x_{0}\right)<f\left(x_{1}\right)$. As a conclusion, there exists $x_{0} \in(0,1)$ such that, for all $x_{0}<x_{1}<x_{2}, f\left(x_{0}\right)<f\left(x_{1}\right)<f\left(x_{2}\right)$. The result is proved.

Several particular cases of the mapping (1) have been addressed in the literature, restricting on particular parametric forms for $f$ :

$$
\begin{aligned}
& f_{\lambda}^{(1)}(x)=1 /(1+\lambda-\lambda x), \lambda \in[0,1], \\
& f_{\lambda}^{(2)}(x)=1-\lambda+\lambda x, \lambda \in[0,1], \\
& f_{\lambda}^{(3)}(x)=\exp (\lambda(x-1)), \lambda \in \mathbb{R}, \\
& f_{\lambda, \theta}^{(4)}(x)=(1+\lambda-\lambda x)^{\theta}, \theta \in \mathbb{R}, \lambda \in[0,1],
\end{aligned}
$$


for all $x \in[0,1]$. In [7], it is proved that $f_{\lambda}^{(1)}$ is admissible while, in [5, Theorem 3.1], it is established that $f_{\lambda}^{(2)}$ is admissible. It is also clear that $f_{\lambda}^{(1)} \in \mathcal{F}$ and $f_{\lambda}^{(2)} \in \mathcal{F}$ which provides an unified proof of the previous results. The next lemma answers some open questions of [5] and extends the result of [7] since $f_{\lambda}^{(1)}=f_{\lambda,-1}^{(4)}$.

Lemma 4 (Examples).

(i) $f_{\lambda}^{(3)}$ is admissible if and only if $\lambda \in[0,1]$. Besides, $f_{\lambda}^{(3)}$ gives rise to the transformed copula introduced in [5], eq. (17).

(ii) $f_{\lambda, \theta}^{(4)}$ is admissible if and only if $\lambda \in[0,1]$ and $-1 / \lambda \leq \theta \leq 0$. Besides, $f_{\lambda, \theta}^{(4)}$ gives rise to a particular case of the transformed copula introduced in [5], eq. (18).

Proof. (i) It is clear that $f_{\lambda}^{(3)} \in \mathcal{D}$ if and only if $\lambda \in[0,1]$. Besides, $\left(f_{\lambda}^{(3)}\right)^{\prime \prime} \geq 0$ for all $\lambda \in \mathbb{R}$ and thus Theorem 1 concludes the proof.

(ii) It is clear that $f_{\lambda, \theta}^{(4)} \in \mathcal{D}$ if and only if $\lambda \in[0,1]$ and $-1 / \lambda \leq \theta \leq 0$. Besides, $\left(f_{\lambda, \theta}^{(4)}\right)^{\prime \prime} \geq 0$ for all $\lambda \in[0,1], \theta \leq 0$ and thus Theorem 1 concludes the proof.

New admissible functions can then be proposed by combining Lemma 3(iii) and Lemma 4. For instance, the function defined for all $x \in[0,1]$ by $f_{\lambda, \theta}^{(5)}(x):=\left(f_{\lambda}^{(3)} \circ f_{1, \theta}^{(4)}\right)(x)=\exp \left(\lambda\left((2-x)^{\theta}-1\right)\right)$ is admissible for all $\lambda \in[0,1]$ and $\theta \in[-1,0]$. The graph of this function is depicted on Figure 1 .

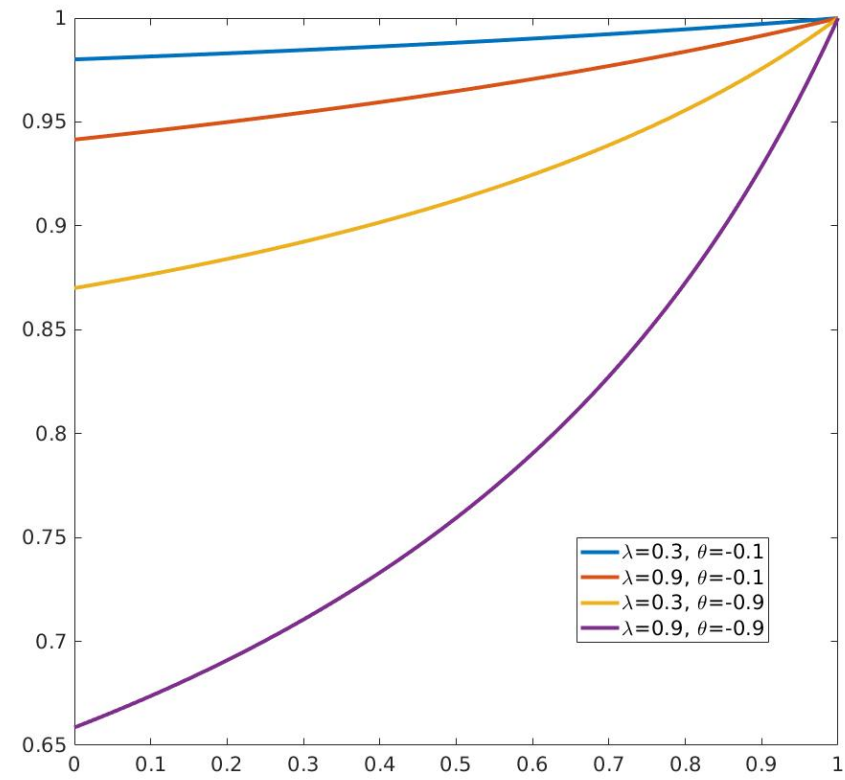

Figure 1: Plot of the function $x \in[0,1] \mapsto f_{\lambda, \theta}^{(5)}(x)=\exp \left(\lambda\left((2-x)^{\theta}-1\right)\right)$ for $\lambda \in\{0.3,0.9\}$ and $\theta \in\{-0.9,-0.1\}$.

\section{Symmetry properties}

Let $(X, Y)$ be a random pair. $X$ and $Y$ are said to be exchangeable if $(X, Y)$ and $(Y, X)$ are identically distributed, see for instance [18], Section 2.7. From [18, Theorem 2.7.4], a necessary and sufficient condition for exchangeability is that $X$ and $Y$ are identically distributed and that their associated copula $C$ is symmetric i.e. $C(u, v)=C(v, u)$ for all $(u, v) \in[0,1]$. The following result is straightforward: 
Proposition 2 (Symmetry). Let $C \in \mathcal{C}$ and $f \in \mathcal{F}$. If $C$ is symmetric then $T(f, C)$ is symmetric.

Let $(a, b) \in \mathbb{R}^{2} .(X, Y)$ is said to be radially symmetric about $(a, b)$ if $(X-a, Y-b)$ and $(a-X, b-Y)$ are identically distributed. Assume that $X$ is symmetric about $a$ and $Y$ is symmetric about $b$. Then, from [18, Theorem 2.7.3], a necessary and sufficient condition for radial symmetry is that

$$
C(u, v)=u+v-1+C(1-u, 1-v) \text { for all }(u, v) \in[0,1]^{2} .
$$

A copula satisfying (9) is said to be radially symmetric. The next result generalizes [5, Theorem 3.9].

Proposition 3 (Radial symmetry). Let $f \in \mathcal{F}$. The copula $T(f, C)$ is radially symmetric for all radially symmetric copula $C$ if and only if $f=f_{\lambda}^{(2)}$.

Proof. From (9), $T(f, C)$ is radially symmetric if and only if, for all $(u, v) \in[0,1]^{2}$ :

$$
u+v-1+C(1-u, 1-v) f(2-u-v-C(1-u, 1-v))=C(u, v) f(u+v-C(u, v)) .
$$

Since $C$ also satisfies (9), the previous equation is equivalent to

$$
u+v-1+(C(u, v)-u-v+1) f(1-C(u, v))=C(u, v) f(u+v-C(u, v)) .
$$

Letting $W_{0}(u, v)=u+v-1$, the previous equation can be rewritten as

$$
W_{0}(u, v)+\left(C(u, v)-W_{0}(u, v)\right) f(1-C(u, v))-C(u, v) f\left(W_{0}(u, v)-C(u, v)+1\right)=0,
$$

or, equivalently, with $\Delta:=f-I_{d}$ :

$$
\frac{\Delta\left(1-\left(C-W_{0}\right)(u, v)\right)}{\left(C-W_{0}\right)(u, v)}=\frac{\Delta(1-C(u, v))}{C(u, v)} .
$$

Finally, introducing $\Psi(\cdot):=\Delta(1-\cdot) / I_{d}(\cdot)$, we end up with the functional equation

$$
\Psi\left(\left(C-W_{0}\right)(u, v)\right)=\Psi(C(u, v)) \text { for all } C \in \mathcal{C} \text { and }(u, v) \in[0,1]^{2} .
$$

Necessarily, $\Psi$ is a constant, say $\lambda$, and therefore $f(t)=(1-\lambda) t+\lambda$. Besides, $f \in \mathcal{F}$ implies $\lambda \in[0,1]$ and the result follows.

\section{Ordering properties}

Let us recall the definition of concordance ordering, see [13], Section 2.2.1 for further details. Let $\left(C_{1}, C_{2}\right) \in \mathrm{C}^{2}$. Then, $C_{1}$ is less concordant than $C_{2}$, written $C_{1} \prec C_{2}$, if $C_{1}(u, v) \leq C_{2}(u, v)$ for all $(u, v) \in[0,1]$.

\section{Proposition 4 (Ordering).}

(i) For all $C \in \mathcal{C}$ and $\left(f_{1}, f_{2}\right) \in \mathcal{F}^{2}, f_{1} \leq f_{2}$ implies $T\left(f_{1}, C\right) \prec T\left(f_{2}, C\right)$.

(ii) For all $f \in \mathcal{F}$, the mapping $T(f, \cdot)$ is increasing with respect to concordance ordering, i.e. $C_{1} \prec C_{2}$ implies $T\left(f, C_{1}\right) \prec T\left(f, C_{2}\right)$.

(iii) $C C^{\star} \prec T(f, C) \prec C$ for all $C \in \mathcal{C}$.

Proof. (i) is straightforward.

(ii) Let $C_{1} \prec C_{2}$ and $f \in \mathcal{F}$. Then, $C_{2}^{\star} \prec C_{1}^{\star}$ implies $f\left(C_{2}^{\star}\right) \geq f\left(C_{1}^{\star}\right) C_{2}^{*} / C_{1}^{\star}$ in view of $I_{d} / f$ is increasing. Routine calculations yield

$$
T\left(f, C_{2}\right)-T\left(f, C_{1}\right) \geq\left(C_{2} C_{2}^{\star}-C_{1} C_{1}^{\star}\right) f\left(C_{1}^{\star}\right) / C_{1}^{\star} .
$$

For all $(u, v) \in[0,1]^{2}$, one has

$$
\left(C_{2} C_{2}^{\star}-C_{1} C_{1}^{\star}\right)(u, v)=\left(C_{2}(u, v)-C_{1}(u, v)\right)\left(u+v-C_{1}(u, v)-C_{2}(u, v)\right)
$$


and $C_{1}(u, v) \leq C_{2}(u, v) \leq \min (u, v) \leq(u+v) / 2$ concludes the proof.

(iii) From Lemma 3(i), $f \in \mathcal{F}$ implies $t \leq f(t) \leq 1$ for all $t \in[0,1]$ and thus Proposition 4(i) yields $T\left(I_{d}, C\right)=$ $C C^{\star} \prec T(f, C) \prec T(1, C)=C$.

Let us now investigate what happens when the transformation $T(f, \cdot)$ is iterated. To this end, let $f \in \mathcal{F}, C \in \mathcal{C}$ and consider the following sequence of transformed copulas:

$$
C_{0}:=C, C_{k+1}=T\left(f, C_{k}\right) \text { for all } k \geq 0 .
$$

The next proposition shows that the iterations converge to the fixed point of $T(f, \cdot)$, which is $W$ the FréchetHoeffding lower bound.

Proposition 5 (Convergence of iterations). Let $C \in \mathcal{C}$ and suppose $f \in \mathcal{F}$ is not identically equal to 1 . Then $C_{k} \rightarrow W$ pointwisely as $k \rightarrow \infty$.

Proof. Let $(u, v) \in[0,1]^{2}$. Clearly $C_{k}(u, v) \geq W(u, v)$ and, from Proposition 4(iii), $C_{k+1}(u, v) \leq C_{k}(u, v)$ for all $k \geq 0$. The sequence $\left(C_{k}(u, v)\right)_{k \geq 1}$ is thus decreasing and lower bounded. As a consequence, this sequence converges to one of the solutions $x=x(u, v)$ of the equation $x f(u+v-x)=x$. Two cases arise: $x(u, v)=0$ or $f(u+v-x)=1$. From Lemma 3(iv), $f(u+v-x)=1$ implies $u+v-x=1$ i.e. $x(u, v)=u+v-1$. Conversely, $x(\cdot, \cdot)$ should be a copula since $\mathcal{C}$ is a closed subset of bivariate functions. Then, $x(\cdot, \cdot)$ should be positive and continuous to define a copula and therefore $x(u, v)=\max (u+v-1,0)$.

The convergence of the sequence $\left(C_{k}\right)$ to $W$ is illustrated on Figure 2 on the simple case where $C=\Pi$ and $f=I_{d}$. The contour plots associated with the densities of $C_{1}, C_{2}, C_{3}$ and $C_{4}$ are depicted. As expected, the contour plots are getting closer to lines with slope -1 as the iterations increase.
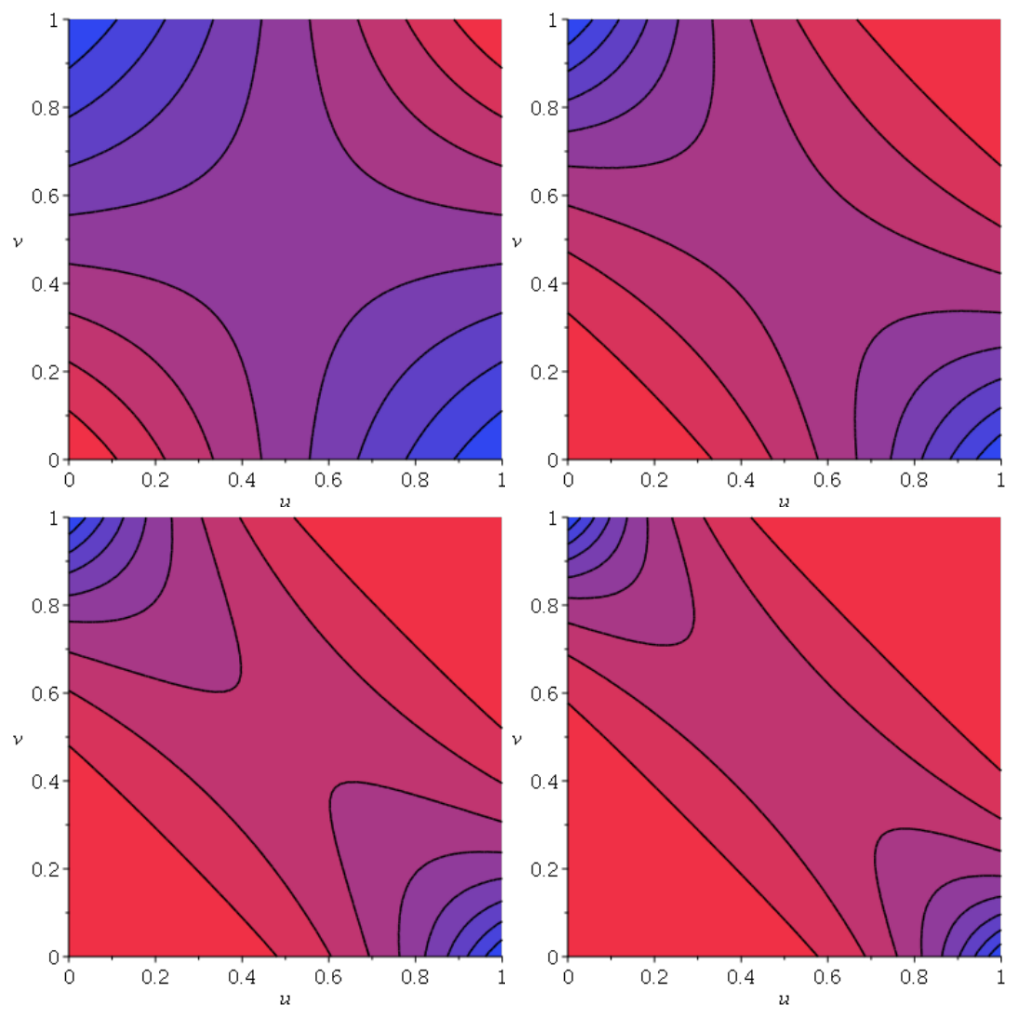

Figure 2: Illustration of the iterated transformation $C_{k+1}=T\left(I_{d}, C_{k}\right)$ of $C_{0}:=\Pi$. From left to right and top to bottom: Contour plots of the densities associated with $C_{1}, \ldots, C_{4}$. Red (resp. blue) color corresponds to small (resp. large) density values. 


\section{Dependence}

Paragraph 5.1 illustrates how the tail dependence coefficients of the copula are modified by the mapping. Some bounds are provided on the measures of association (Spearman's Rho and Kendall's Tau) of the transformed copula in Paragraph 5.2. Finally, it is shown in Paragraph 5.3 that the mapping preserves dependence properties such as Negative Quadrant Dependence property, Left Tail Increasing property and Stochastic Decreasing property.

\subsection{Measures of tail dependence}

The tail dependence coefficients are defined as the conditional probabilities that a random vector associated with a copula $C$ belongs to lower or upper tail orthants given that a univariate margin takes extreme values [21]:

$$
\begin{array}{cc}
\lambda_{L L}(C)=\lim _{u \rightarrow 0^{+}} \frac{C(u, u)}{u}, & \lambda_{U L}(C)=1-\lim _{u \rightarrow 0^{+}} \frac{C(1-u, u)}{u}, \\
\lambda_{L U}(C)=1-\lim _{u \rightarrow 0^{+}} \frac{C(u, 1-u)}{u}, & \lambda_{U U}(C)=2-\lim _{u \rightarrow 1^{-}} \frac{C(u, u)-1}{u-1} .
\end{array}
$$

The next proposition establishes that $\lambda_{U L}$ and $\lambda_{L U}$ are left invariant by the mapping while $\lambda_{L L}$ and $\lambda_{U U}$ may be decreased, depending on $f(0)$ and $f^{\prime}\left(1^{-}\right)$respectively. Let us highlight that $f^{\prime}\left(1^{-}\right)$exists for all $f \in \mathcal{F}$ since, in this case, $f$ is continuous and convex.

Proposition 6 (Tail dependence coefficients). Let $C \in \mathcal{C}, f \in \mathcal{F}$. Then,

$$
\begin{aligned}
\lambda_{L L}(T(f, C)) & =\lambda_{L L}(C) f(0) \\
\lambda_{U L}(T(f, C)) & =\lambda_{U L}(C) \\
\lambda_{L U}(T(f, C)) & =\lambda_{L U}(C) \\
\lambda_{U U}(T(f, C)) & =\lambda_{U U}(C)\left(1-f^{\prime}\left(1^{-}\right)\right) .
\end{aligned}
$$

Proof. First,

$$
\lambda_{L L}(T(f, C))=\lim _{u \rightarrow 0^{+}} \frac{T(f, C)(u, u)}{u}=\lim _{u \rightarrow 0^{+}} \frac{C(u, u)}{u} f(2 u-C(u, u))=\lambda_{L L}(C) f(0) .
$$

Similarly,

$$
1-\lambda_{U L}(T(f, C))=\lim _{u \rightarrow 0^{+}} \frac{T(f, C)(1-u, u)}{u}=\lim _{u \rightarrow 0^{+}} \frac{C(1-u, u)}{u} f(1-C(1-u, u))=\left(1-\lambda_{U L}(C)\right) f(1),
$$

and recalling that $f(1)=1$ gives the result. The calculation of $\lambda_{L U}(T(f, C))$ follows the same lines. Finally, let us consider $\lambda_{U U}(T(f, C))$. A first order Taylor expansion as $u \rightarrow 1^{-}$yields

$$
f(2 u-C(u, u))=1+(2 u-C(u, u)-1) f^{\prime}\left(1^{-}\right)+o(u-1)
$$

and therefore

$$
\begin{aligned}
\frac{T(f, C)(u, u)-1}{u-1} & =\frac{C(u, u) f(2 u-C(u, u)-1)}{u-1} \\
& =\frac{C(u, u)-1}{u-1}+C(u, u) f^{\prime}\left(1^{-}\right) \frac{2 u-C(u, u)-1}{u-1}+o(1)
\end{aligned}
$$

Taking the limit as $u \rightarrow 1^{-}$yields

$$
2-\lambda_{U U}(T(f, C))=2-\lambda_{U U}(C)+f^{\prime}\left(1^{-}\right) \lambda_{U U}(C),
$$

and the result follows. 


\subsection{Measures of association}

Several measures of association between the components of a random pair $(X, Y)$ can be considered: the normalized volume [19], Kendall's Tau [18], Paragraph 5.1.1, Gini's gamma [12], Blomqwist's medial correlation coefficient [18], Paragraph 5.1.4, Spearman's footrule [12] and Spearman's Rho [18], Paragraph 5.1.2. All these measures are invariant to strictly increasing functions.

The medial correlation coefficient, introduced by Blomqvist [2], is defined as

$$
\beta=\mathbb{P}((X-\tilde{x})(Y-\tilde{y})>0)-\mathbb{P}((X-\tilde{x})(Y-\tilde{y})<0)
$$

where $\tilde{x}$ and $\tilde{y}$ are the medians on the random variables $X$ and $Y$ respectively. In case of continuous random variables, $\beta$ depends only on the associated copula: $\beta=\beta(C)=4 C(1 / 2,1 / 2)-1$, see [18], equation (5.1.27). The next result shows how the medial correlation coefficient is modified by the mapping $T(\cdot, f)$.

Proposition 7 (Medial correlation coefficient). Let $C \in \mathcal{C}, f \in \mathcal{F}$. Then,

$$
\beta(T(f, C))=(\beta(C)+1) f((3-\beta(C)) / 4)-1 .
$$

The proof is straightforward. Kendall's Tau and Spearman's Rho can be interpreted as probabilities of concordance minus probabilities of discordance of two random pairs: Kendall's Tau is the probability of concordance minus the probability of discordance of two identically distributed random pairs $\left(X_{1}, Y_{1}\right)$ and $\left(X_{2}, Y_{2}\right)$. Spearman's Rho is the probability of concordance minus the probability of discordance of two pairs $\left(X_{1}, Y_{1}\right)$ and $\left(X_{2}, Y_{2}\right)$ with respective copulas $C$ and $\Pi$. In case of continuous random variables, both of them can be rewritten in terms of copulas, for instance:

$$
\rho=\rho(C)=12 \int_{0}^{1} \int_{0}^{1} C(u, v) d u d v-3 .
$$

Note that $\rho$ coincides with the correlation coefficient between the uniform marginal distributions. Obtaining a result similar to Proposition 7 for Spearman's Rho or Kendall's Tau seems a difficult task. It is however clear that the following (sharp) bounds hold.

Proposition 8 (Spearman's Rho and Kendall's Tau).

(i) Let $C \in$ C. For all $f \in \mathcal{F}$ :

$$
\rho\left(C C^{\star}\right) \leq \rho(T(f, C)) \leq \rho(C) \text { and } \tau\left(C C^{\star}\right) \leq \tau(T(f, C)) \leq \tau(C) .
$$

(ii) Let $f \in \mathcal{F}$. For all $C \in \mathcal{C}$ :

$$
-1 \leq \rho(T(f, C)) \leq 12 \int_{0}^{1} x^{2} f(x) d x-3 \text { and }-1 \leq \tau(T(f, C)) \leq 4 \int_{0}^{1} x f^{2}(x) d x-1 .
$$

Proof. (i) is a consequence of Proposition 4(iii).

(ii) In view of Proposition 4(ii) and Lemma $2, W=T(f, W) \prec T(f, C) \prec T(f, M)=C_{f}$. As a consequence, $-1=\rho(W) \leq \rho(T(f, C)) \leq \rho\left(C_{f}\right)=12 \int_{0}^{1} x^{2} f(x) d x-3$ from [6, Proposition 3.4].

The proof is similar for Kendall's $\tau$.

\subsection{Dependence properties}

Let $(X, Y)$ be a pair of random variables with continuous margins and associated copula $C$.

- $\quad X$ and $Y$ are said to be Negatively Quadrant Dependent (NQD) if

$$
\mathbb{P}(X \leq x, Y \leq y) \leq \mathbb{P}(X \leq x) \mathbb{P}(Y \leq y) \text { for all }(x, y) .
$$


Since this property can be characterized by the copula property $C \prec \Pi$, see for instance [18], Paragraph 5.2.1, we shall write for short that $C$ is NQD.

- $X$ and $Y$ are said to be Left Tail Increasing (LTI) if $\mathbb{P}(X \leq x \mid Y \leq y)$ is a nondecreasing function of $y$ for all $x$, and $\mathbb{P}(Y \leq y \mid X \leq x)$ is a nondecreasing function of $x$ for all $y$. From [18, Theorem 5.2.5], this property can be characterized by the copula properties

$$
\begin{gathered}
C(u, v) / u \text { is nondecreasing in } u \text { for all } v \in[0,1], \\
C(u, v) / v \text { is nondecreasing in } v \text { for all } u \in[0,1],
\end{gathered}
$$

and we shall thus write that $C$ is LTI.

- $\quad X$ and $Y$ are said to be Stochastically Decreasing (SD) if $\mathbb{P}(X>x \mid Y=y)$ is a nonincreasing function of $y$ for all $x$, and $\mathbb{P}(Y>y \mid X=x)$ is a nonincreasing function of $x$ for all $y$. From [18, Corollary 5.2.11], this property can be characterized by the copula properties

$$
\begin{aligned}
& C(u, v) \text { is a convex function of } u \text { for all } v \in[0,1], \\
& C(u, v) \text { is a convex function of } v \text { for all } u \in[0,1],
\end{aligned}
$$

and we shall thus write that $C$ is SD.

The three properties NQD, LTI and SD are preserved by the mapping.

Proposition 9 (Dependence properties). Let $f \in \mathcal{F}$ and $C \in \mathcal{C}$.

(i) If $C$ is $N Q D$ then $T(f, C)$ is $N Q D$.

(ii) If $C$ is LTI then $T(f, C)$ is LTI.

(iii) If $C$ is $S D$ then $T(f, C)$ is $S D$.

Proof. (i) Proposition 4(ii,iii) show that $C \prec \Pi$ implies $T(f, C) \prec T(f, \Pi) \prec \Pi$. The result is proved.

(ii) Let $(u, v) \in[0,1]^{2}$. Our goal is to prove that $T(f, C)(u, v) / u$ is nondecreasing in $u$ for all $v$, the study of $T(f, C)(u, v) / v$ being similar Assume that $C$ is an absolutely continuous copula with continuous partial derivatives and that $f$ is differentiable:

$$
\begin{aligned}
u^{2} \frac{\partial T(f, C)(u, v) / u}{\partial u} & =u D_{1} T(f, C)(u, v)-T(f, C)(u, v) \\
& =f\left(C^{\star}(u, v)\right)\left(u D_{1} C(u, v)-C(u, v)\right) \\
& +u C(u, v) f^{\prime}\left(C^{\star}(u, v)\right)\left(1-D_{1} C(u, v)\right) .
\end{aligned}
$$

From (10), it follows that (12) is nonnegative. Besides, [18, Theorem 2.2.7] entails that (13) is nonnegative and the result is proved. The case of an arbitrary copula $C$ in $\mathcal{C}$ and arbitrary function $f$ in $\mathcal{F}$ is addressed with density arguments, see the proof of Theorem 1.

(iii) Let $(u, v) \in[0,1]^{2}$. Our goal is to prove that $T(f, C)(\cdot, v)$ is a convex function for all $v$, the study of $T(f, C)(u, \cdot)$ being similar. Assume that $C$ is an absolutely continuous copula with continuous partial derivatives and that $f$ is twice differentiable:

$$
\begin{aligned}
D_{11} T(f, C) & =D_{11} C\left[f\left(C^{\star}\right)-C f^{\prime}\left(C^{\star}\right)\right] \\
& +2 D_{1} C f^{\prime}\left(C^{\star}\right)\left(1-D_{1} C\right) \\
& +C f^{\prime \prime}\left(C^{\star}\right)\left(1-D_{1} C\right)^{2} .
\end{aligned}
$$

From (11), it follows that $D_{11} C$ is nonnegative. Besides, $f\left(C^{\star}\right)-C f^{\prime}\left(C^{\star}\right) \geq\left(C^{\star}-C\right) f^{\prime}\left(C^{\star}\right)$ as already seen if the proof of Theorem 1 , and

$$
\left(C^{\star}-C\right)(u, v)=u+v-2 C(u, v) \geq u+v-2 \min (u, v)=\max (u, v)-\min (u, v) \geq 0 .
$$

If follows that (14) is nonnegative. Besides, [18, Theorem 2.2.7] entails that (15) is nonnegative. Finally, (16) is nonnegative since $f \in \mathcal{F}$, and the result follows. The case of an arbitrary copula $C$ in $\mathcal{C}$ and arbitrary function $f$ in $\mathcal{F}$ is addressed as in the proof of (ii). 
Acknowledgement: The author warmly thanks an anonymous referee for his/her helpful suggestions to establish the necessary condition in Theorem 1.

\section{References}

[1] Amblard, C. and S. Girard (2009). A new extension of bivariate FGM copulas. Metrika 70(1), 1-17.

[2] Blomqvist, N. (1950). On a measure of dependence between two random variables. Ann. Math. Statist. 21(4), 593-600.

[3] Bruckner, A.M. and E. Ostrow (1962). Some function classes related to the class of convex functions. Pacific J. Math 12(4), 1203-1215.

[4] Di Bernardino, E. and D. Rullière (2013). On certain transformations of Archimedean copulas: Application to the nonparametric estimation of their generators. Depend. Model. 1,1-36.

[5] Dolati, A. and M. Úbeda-Flores (2009). Constructing copulas by means of pairs of order statistics. Kybernetika 45(6), 9921002.

[6] Durante, F. (2006). A new class of symmetric bivariate copulas. J. Nonparametr. Stat. 18(7-8), 499-510.

[7] Durante, F., J. Fernández-Sánchez, and W. Trutschnig (2014). Solution to an open problem about a transformation on the space of copulas. Depend. Model. 2, 65-72.

[8] Durante, F., R. Foschi, and P. Sarkoci (2010). Distorted copulas: constructions and tail dependence. Comm. Statist. Theory Methods 39(12), 2288-2301.

[9] Durante, F., S. Girard, and G. Mazo (2015). Copulas based on Marshall-Olkin machinery. In U. Cherubini, F. Durante, and S. Mulinacci (Eds.), Marshall-Olkin Distributions - Advances in Theory and Applications, pp. 15-31. Springer, Cham.

[10] Durante, F., S. Girard, and G. Mazo (2016). Marshall-Olkin type copulas generated by a global shock. J. Comput. Appl. Math. 296, 638-648.

[11] Durante, F. and C. Sempi (2016). Principles of Copula Theory. CRC Press, Boca Raton FL.

[12] Genest, C., J. Nešlehovà, and N. B. Ghorbal (2010). Spearman's footrule and Gini's gamma: a review with complements. J. Nonparametr. Stat. 22(8), 937-954.

[13] Joe, H. (1997). Multivariate Models and Dependence Concepts. Chapman \& Hall, London.

[14] Klement, E. P., R. Mesiar, and E. Pap (2005). Transformations of copulas. Kybernetika 41(4), 425-434.

[15] Manstavicius, M. and G. Bagdonas (2019). A class of bivariate copula mappings. Fuzzy Sets Syst., 354(1), 48-62.

[16] Michiels, F. and A. De Schepper (2012). How to improve the fit of Archimedean copulas by means of transforms. Stat. Papers 53(2), 345-355.

[17] Morillas, P. M. (2005). A method to obtain new copulas from a given one. Metrika 61(2), 169-184.

[18] Nelsen, R. B. (2006). An Introduction to Copulas. Second edition. Springer, New York.

[19] Schweizer, B. and E. F. Wolff (1981). On nonparametric measures of dependence for random variables. Ann. Statist. 9(4), 879-885.

[20] Valdez, E. A. and Y. Xiao (2011). On the distortion of a copula and its margins. Scand. Actuar. J. 2011(4), 292-317.

[21] Zhang, M.-H. (2008). Modelling total tail dependence along diagonals. Insurance Math. Econom. 42(1), 73-80. 